\title{
Perspective Treating Mouse Models of Huntington Disease
}

\author{
Sara J Tallaksen-Greene' and Roger L Albin*,1,2 \\ 'Department of Neurology, University of Michigan, Ann Arbor, MI, USA; ${ }^{2}$ Geriatrics Research, Education, and Clinical Center, VAAAHS, Ann Arbor, \\ MI, USA
}

Neuropsychopharmacology (20 I I) 36, 2373-2374; do::I0.1038/npp.20 I I.I58; published online 3 August 20I I

In this issue of Neuropsychopharmacology, Chiu et al (2011) present preclinical data that combined administration of two widely used drugs, lithium $(\mathrm{Li})$ and valproate acid (VPA), which therapeutic effects in two murine genetic models of Huntington disease (HD). HD is a dominantly inherited, adult-onset neurodegenerative disorder with prominent psychiatric manifestations. It is presently untreatable and uniformly fatal. Although uncommon, HD is an attractive target for neuroprotective therapy because a single type of mutation, expanded CAG repeats within the open reading frame of the huntingtin (htt) gene, causes all cases of $\mathrm{HD}$, mutant alleles exhibit $100 \%$ penetrance, and presymptomatic carriers can be identified easily. Although $h t t$ and the characteristic mutation were discovered almost 20 years ago, the normal function of $h t t$ remains elusive. Research over the past couple of decades provides creditable evidence for several proximate mechanisms of neurodegeneration driven by mutant $h t t$. This latter fact presents a real challenge to the development of effective therapies, as it is unlikely that targeting a single mechanism of neurodegeneration will be effective. This is a solid rationale for some form of polytherapy.

Chiu et al (2011) present a well-performed study of combination therapy with Li and VPA in preclinical HD mouse models. Distinguishing features of this study include positive results in two different models, favorable outcomes in several behavioral measures, reduction of mortality in one model, and biochemical biomarker evidence of relevant effects in brain. As Li and VPA are widely used agents in psychiatric and neurologic practice, the authors reasonably suggest that this combination should be pursued in neuroprotective clinical trials for HD.

This study also highlights some of the major challenges in HD research. How valid are these animal models? Both are transgenic models. One, N171-82Q, expresses a htt fragment containing the expanded polyCAG domain. The second, YAC128, expresses full-length human $h t t$. Both these models exhibit good face validity in that they produce some

*Correspondence: Dr RL Albin, Department of Neurology, University of Michigan, 5023 BSRB, 109 Zina Pitcher Place, Ann Arbor, MI 48I092200, USA, Tel: + I 734764 |347, Fax: + I 734763 7686,

E-mail: ralbin@umich.edu

Received 7 July 201 I; accepted 8 July 201 I reasonable analogs of the HD behavioral phenotype and HD pathology. Other features are somewhat discordant. Manifest HD usually begins in middle age and N171-82Q mice have a very early onset phenotype. The YAC128 phenotype appears to plateau in mid-life, while HD is inexorably progressive. In terms of construct validity, both express the core feature of expanded polyCAG repeats. Although the CAG repeat expansion is the primary vector of neurodegeneration, there is considerable evidence that the surrounding protein domains strongly influence the mechanisms of neurodegeneration. Fragment models like N171-82Q may not be faithful mimics of HD. The YAC128 does express the whole human protein but not in its normal genomic regulatory context, which could be significant. Perhaps most important, the predictive validity of these and all other models of HD is unknown. Only one substantial phase 3 trial has been completed in HD and there is little human clinical trial data for model validation. Until further relevant clinical research is completed, the value of animal models is uncertain.

These experiments point also to another potential problem in developing treatments for HD. Chiu et al (2011) found that very early treatment of N171-82Q mice was necessary to obtain a positive effect on mortality. If extrapolated to humans, this would be a major problem for clinical trials and for actual clinical application. The median age of onset of manifest HD is around 40, though manifest $\mathrm{HD}$ is defined by the presence of a movement disorder and many patients have behavioral features years in advance of the development of movement disorders. Neuroimaging data suggest that the brain changes begin to occur in the early $20 \mathrm{~s}$. Would a trial of Li and VPA have to be initiated in the early 20s and patients followed for decades? This would be formidably difficult. In addition, the acceptable standard for a prophylactic treatment is somewhat different than that for treating a symptomatic population. As prophylactic treatments are applied in asymptomatic individuals, prophylactic treatments have to possess highly favorable therapeutic indices. Agents with significant side effect rates are likely to lead to high dropout rate in trials and may be ineffective in clinical practice because of poor patient compliance. As the authors acknowledge, Li has a narrow therapeutic window. VPA is generally easier to use in practice but also has features which would be an obstacle to 
clinical use in this setting. VPA, for example, has significant teratogenic potential. For prophylactic treatment of $\mathrm{HD}$, it would have to be prescribed to women of child-bearing age.

All these facts underscore the difficulties of translating potential therapies from the laboratory to the clinic. This appears to be particularly difficult for neurodegenerative disorders.

\section{ACKNOWLEDGEMENTS}

This study was supported by a VA Merit Review grant.

\section{DISCLOSURE}

Dr Albin has received compensation for acting as an expert witness in litigation concerning dopamine agonist-induced impulse control disorders. Dr Tallaksen-Greene declares no conflict of interest.

\section{REFERENCES}

Chiu C-T, Liu G, Leeds P, Chuang D-M (2011). Combined treatment with the mood stabilizers lithium and valproate produces multiple beneficial effects in transgenic mouse models of Huntington's disease. Neuropsychopharmacology 36: 2406-2421. 